\title{
Independence of Local Algebras in Quantum Field Theory
}

\author{
H. Roos \\ Institut für Theoretische Physik der Universität Göttingen
}

Received October 15, 1969

\begin{abstract}
It is shown that local $C^{*}$-algebras $\mathfrak{A}\left(O_{1}\right)$ and $\mathfrak{A}\left(O_{2}\right)$ associated with spacelike separated regions $O_{1}$ and $O_{2}$ in the Minkowski space are independent. The proof is accomplished by a theorem concerning the structure of the $C^{*}$-algebra generated by $\mathfrak{U}\left(O_{1}\right)$ and $\mathfrak{A}\left(O_{2}\right)$.
\end{abstract}

\section{Introduction}

Let $\mathfrak{A}, \mathfrak{A}_{1}, \mathfrak{A}_{2}$ be $C^{*}$-algebras with $\mathfrak{A}_{1}$ and $\mathfrak{A}_{2}$ contained in $\mathfrak{A}$. Picking a state $\varphi_{1}$ of $\mathfrak{U}_{1}$ and a state $\varphi_{2}$ of $\mathfrak{U}_{2}$ one may ask whether there exists a state $\varphi$ of $\mathfrak{A}$ whose restriction to $\mathfrak{A}_{i}$ equals $\varphi_{i}(i=1,2)$. If this is the case for any choice of the pair $\varphi_{1}, \varphi_{2}$ then we shall say that the algebras $\mathfrak{X}_{1}$ and $\mathfrak{U}_{2}$ are "statistically independent".

In a Quantum Field Theory let $\mathfrak{A}(O)$ denote the algebra of observables which are associated with the region $O$ of the Minkowski space. We use the symbol $O_{1} \times O_{2}$ to denote that two regions $O_{1}, O_{2}$ lie totally spacelike to each other. In [1] Haag and Kastler raised the question of whether two algebras $\mathfrak{H}\left(O_{1}\right)$ and $\mathfrak{H}\left(O_{2}\right)$ are statistically independent when $O_{1} \times O_{2}$.

If $O_{1}+x \times O_{2}$ for $x \in \mathscr{N}, \mathscr{N}$ being a suitably chosen neighbourhood of the origin, we write $O_{1} * O_{2}$. Starting from standard assumptions of Quantum Field Theory, Schlieder [2] derived the following

Proposition (Schlieder). Suppose $O_{1} \nsim O_{2}$. If $x \in \mathfrak{A}\left(O_{1}\right)$ and $y \in \mathfrak{A}\left(O_{2}\right)$ are non-vanishing elements, then $x y \neq 0$.

Schlieder also pointed out that the property $x y \neq 0$ for non-vanishing pairs of elements of two commuting algebras $\mathfrak{A}_{1}, \mathfrak{A}_{2}$ is a necessary condition for statistical independence. We shall show here that this property is also a sufficient condition. One has

Theorem 1. Let $\mathfrak{A}, \mathfrak{I}_{1}, \mathfrak{A}_{2}$ be $C^{*}$-algebras with unit elements and let $\mathfrak{U}_{i} \subset \mathfrak{A}$. 


\section{Suppose}

$(C): \mathfrak{A}_{1}$ and $\mathfrak{A}_{2}$ commute elementwise.

Then $\mathfrak{\mathfrak { A }}_{1}$ and $\mathfrak{\mathfrak { H }}_{2}$ are statistically independent if and only if they have the property $(S)$ : If $x$ and $y$ are non-vanishing elements of $\mathfrak{U}_{1}$ and $\mathfrak{U}_{2}$ respectively, then $x y \neq 0$.

In addition, we shall show

Proposition 1. Let $\mathfrak{I}_{1}$ and $\mathfrak{A}_{2}$ be statistically independent, $\mathfrak{A}_{1}$ and $\mathfrak{A}_{2}$ commuting, $\mathfrak{H}_{i} \subset \mathfrak{H}$. If $\varphi_{1}$ is a pure state over $\mathfrak{X}_{1}$ and $\varphi_{2}$ is a pure state over $\mathfrak{H}_{2}$, then there exists an extension $\varphi$ of $\varphi_{1}$ and $\varphi_{2}$ which is a pure state over $\mathfrak{A}$.

II.

In this section and in the following one, we shall prove some lemmas and another theorem which will finally yield the proofs of Theorem 1 and Proposition 1. The first essential step is the demonstration of the following

Lemma 1. Let $\mathfrak{U}, \mathfrak{U}_{1}, \mathfrak{A}_{2}$ be as in Theorem 1, satisfying $(C)$ and $(S)$. Suppose $\sum_{i=1}^{n} x_{i} y_{i}=0$ with $x_{i} \in \mathfrak{A}_{1}, y_{i} \in \mathfrak{A}_{2}$. Then, unless all $x_{i}=0$ or all $y_{i}=0$, neither the $\left\{x_{i}, i=1, \ldots n\right\}$ nor the $\left\{y_{i}, i=1, \ldots n\right\}$ can be linearly independent.

We need another lemma to prove this. Let $\mathfrak{B}_{i}$ be an abelian $C^{*}$ subalgebra of $\mathfrak{A}_{i}, i=1,2$; let $\mathfrak{B}_{i}^{*}$ be its spectrum, that is, the set of all characters of $\mathfrak{B}_{i}$ with the weak topology [3]. The elements of $\mathfrak{B}_{1}^{*}$ and $\mathfrak{B}_{2}^{*}$ may be denoted by $\chi^{\prime}$ and $\chi^{\prime \prime}$ respectively. Since $\mathfrak{B}_{1}$ and $\mathfrak{B}_{2}$ commute, they generate an abelian $C^{*}$-subalgebra $\mathfrak{B}_{12}$ of $\mathfrak{U}, \mathfrak{B}_{12}^{*}$ denoting its spectrum. A character $\chi \in \mathfrak{B}_{12}^{*}$, restricted to $\mathfrak{B}_{i}$, clearly defines an element of $\mathfrak{B}_{i}^{*}: \chi \mid \mathfrak{B}_{i} \in \mathfrak{B}_{i}^{*}$. Now define the subset $\mathscr{M}$ of the topological product $\mathfrak{B}_{1}^{*} \times \mathfrak{B}_{2}^{*}$ by

$$
\mathscr{M}=\left\{\left(\chi\left|\mathfrak{B}_{1}, \chi\right| \mathfrak{B}_{2}\right) \mid \chi \in \mathfrak{B}_{12}^{*}\right\} .
$$

Lemma 2. If $(S)$ is satisfied, then $\mathscr{M}$ is dense in $\mathfrak{B}_{1}^{*} \times \mathfrak{B}_{2}^{*}$.

Proof. Assume the contrary. Then we can find an element $\left(\chi_{0}^{\prime}, \chi_{0}^{\prime \prime}\right)$ and a neighbourhood $U\left(\left(\chi_{0}^{\prime}, \chi_{0}^{\prime \prime}\right)\right)$ such that $\mathscr{M} \cap U=\emptyset$. $U$ contains a neighbourhood $U_{1}\left(\chi_{0}^{\prime}\right) \times U_{2}\left(\chi_{0}^{\prime \prime}\right)$. Define continuous functions $f\left(\chi^{\prime}\right)$ and $g\left(\chi^{\prime \prime}\right)$ over $\mathfrak{B}_{1}$ and $\mathfrak{B}_{2}$ respectively, with $\operatorname{supp} f \subset U_{1}, \operatorname{supp} g \subset U_{2}$. As is well known, $\mathfrak{B}_{i}$ is isomorphic to the $C^{*}$-algebra of continuous complex functions over $\mathfrak{B}_{i}^{*}$ vanishing at infinity; the isomorphism is furnished by the Gelfand transformation ([4], Theorem 1.4.1). Therefore, if $f$ and $g$ do not vanish identically, they are Gelfand transforms of elements 
$x \in \mathfrak{B}_{1}$ and $y \in \mathfrak{B}_{2}$. Consider $\chi(x y)$ for arbitrary $\chi \in \mathfrak{B}_{12}^{*}$. Clearly,

$$
\chi(x y)=\chi(x) \chi(y)=f\left(\chi \mid \mathfrak{B}_{1}\right) g\left(\chi \mid \mathfrak{B}_{2}\right)=0
$$

because of our assumption $\mathscr{M} \cap U=\emptyset$ and the support properties of $f$ and $g$. Hence $x y=0, x \neq 0, y \neq 0$, which contradicts the property $(S)$.

Proof of Lemma 1. (i) The main task is to prove the lemma for commuting $x_{i}$ and commuting $y_{i}$. Let $\mathfrak{B}_{1}$ and $\mathfrak{B}_{2}$ be abelian $C^{*}$-subalgebras of $\mathfrak{A}_{1}$ and $\mathfrak{A}_{2}$ containing $\left\{x_{i}\right\}$ and $\left\{y_{i}\right\}$ respectively. $\sum_{i=1}^{n} x_{i} y_{i}=0$ implies

$$
\chi\left(\sum_{i=1}^{n} x_{i} y_{i}\right)=\sum_{i=1}^{n} \chi\left(x_{i}\right) \chi\left(y_{i}\right)=\sum_{i=1}^{n} \chi\left|\mathfrak{B}_{1}\left(x_{i}\right) \chi\right| \mathfrak{B}_{2}\left(y_{i}\right)=0
$$

for all $\chi \in \mathfrak{B}_{12}^{*}$ and, with the help of Lemma 2,

$$
\sum_{i=1}^{n} \chi^{\prime}\left(x_{i}\right) \chi^{\prime \prime}\left(y_{i}\right)=0 \quad \text { for all } \quad \chi^{\prime} \in \mathfrak{B}_{1}^{*}, \chi^{\prime \prime} \in \mathfrak{B}_{2}^{*} .
$$

Unless all $y_{i}=0$, we can find a $\chi_{0}^{\prime \prime}$ such that not all $\chi_{0}^{\prime \prime}\left(y_{i}\right)$ vanish. With $\gamma_{i}=\chi_{0}^{\prime \prime}\left(y_{i}\right)$ we have

$$
\chi^{\prime}\left(\sum \gamma_{i} x_{i}\right)=\sum \chi^{\prime}\left(x_{i}\right) \chi_{0}^{\prime \prime}\left(y_{i}\right)=0 \text { for all } \chi^{\prime} \in \mathfrak{B}_{1}^{*},
$$

and therefore, $\sum \gamma_{i} x_{i}=0$. Due to the symmetry of Eq. (1) with respect to $\left\{x_{i}\right\}$ and $\left\{y_{i}\right\}$, the $\left\{y_{i}\right\}$ are linearly dependent, too.

(ii) Now let us consider $x_{i}, y_{i}$ which do not all commute with each other, with $\sum_{i=1}^{n} x_{i} y_{i}=0$. Without loss of generality, we may assume that there exists a $y_{k_{0}}$ such that not all $y_{i}^{\prime}=\left[y_{i}, y_{k_{0}}\right]$ vanish, and we have

$$
\sum_{\substack{i=1 \\ i \neq k_{0}}}^{n} x_{i} y_{i}^{\prime}=0 .
$$

Trivially, the lemma is true for $n=1$. Suppose it is proven for $v \leqq n-1$. Because the sum in (2) contains less than $n$ terms, the $\left\{x_{i}, i \neq k_{0}\right\}$ and, of course, the $\left\{x_{i}, i=1, \ldots n\right\}$ are linearly dependent. Let $\gamma_{i_{0}} \neq 0, c_{i}=\gamma_{i} / \gamma_{i_{0}}$, $x_{i_{0}}=-\sum_{i \neq i_{0}} c_{i} x_{i}$. It follows that $\sum_{i \neq i_{0}} x_{i}\left(y_{i}-c_{i} y_{i_{0}}\right)=0$. Then either all $y_{i}=c_{i} y_{i_{0}}$, which gives us already the desired linear dependence of the $\left\{y_{i}\right\}$ or not all $\left(y_{i}-c_{i} y_{i_{0}}\right)=0$; and therefore, since we have less than $n$ terms, we can find non-trivial $\beta_{i}$ with

$$
\sum_{i \neq i_{0}} \beta_{i} y_{i}+\left(\sum_{i \neq i_{0}} \beta_{i} c_{i}\right) y_{i_{0}}=\sum_{i \neq i_{0}} \beta_{i}\left(y_{i}-c_{i} y_{i_{0}}\right)=0 .
$$

This proves Lemma 1 [5].

Now it is easy to demonstrate 
Proposition 2. Let $\mathfrak{A}, \mathfrak{U}_{1}, \mathfrak{A}_{2}$ be as in Lemma 1, satisfying (C) and (S). Suppose $\sum_{i=1}^{n} x_{i} y_{i}=0, x_{i} \in \mathfrak{A}_{1}, y_{i} \in \mathfrak{A}_{2}$, not all $x_{i}=0$, not all $y_{i}=0$. Then there exist non-trivial complex numbers $\alpha_{i k}$ such that

$$
\begin{aligned}
& \sum_{i=1}^{n} \alpha_{i k} x_{i}=0, \quad k=1, \ldots n, \\
& \sum_{k=1}^{n} \alpha_{i k} y_{k}=y_{i}, \quad i=1, \ldots n .
\end{aligned}
$$

$\alpha_{i k}$ are called non-trivial if

1) not all $\alpha_{i k}$ vanish,

2) not all $\alpha_{i k}=\delta_{i k}=\left\{\begin{array}{ll}0, & i \neq k \\ 1, & i=k\end{array}\right.$.

Proposition 2 is so to speak symmetric in $\left\{x_{i}\right\}$ and $\left\{y_{i}\right\}$ because with $\alpha_{i k}^{\prime}=-\alpha_{k i}+\delta_{k i}$ we have

$$
\sum_{i} \alpha_{i k}^{\prime} y_{i}=0, \quad k=1, \ldots n ; \quad \sum_{k} \alpha_{i k}^{\prime} x_{k}=x_{i}, \quad i=1, \ldots n,
$$

with non-trivial $\alpha_{i k}^{\prime}$.

Proof by induction. $n=1$ is evident due to assumption $(S)$. Let the assertion be proven for $v \leqq n-1 . v=n$ : According to Lemma $1,\left\{x_{i}\right\}$ are linearly dependent; without loss of generality, let us assume that $x_{1}=-\sum_{i=2}^{n} \gamma_{i} x_{i}$. This implies $\sum_{i=2}^{n} x_{i}\left(y_{i}-\gamma_{i} y_{1}\right)=0$. If not all $y_{i}=\gamma_{i} y_{1}$, there exist non-trivial numbers $\beta_{i k}$ with $\sum_{i=2}^{n} \beta_{i k} x_{i}=0, \quad k=2, \ldots n ; \quad \sum_{k=2}^{n} \beta_{i k}\left(y_{k}-\gamma_{k} y_{1}\right)=y_{i}-\gamma_{i} y_{1}, \quad i=2, \ldots n$, since we assume that the proposition is true for $v \leqq n-1$. If one puts

$$
\begin{aligned}
\alpha_{11} & =1, \\
\alpha_{1 k} & =0, \quad k=2, \ldots n, \\
\alpha_{i 1} & =\gamma_{i}-\sum_{k=2}^{n} \beta_{i k} \gamma_{k}, \quad i=2, \ldots n, \\
\alpha_{i k} & =\beta_{i k}, \quad i, k \geqq 2,
\end{aligned}
$$

one can directly verify that Eqs. (3) and (4) hold. Clearly, $\alpha_{i k}$ are nontrivial because $\beta_{i k}$ are non-trivial. If $y_{i}=\gamma_{i} y_{1}$ for all $i=2, \ldots n$, then $\left(x_{1}+\sum_{i=2}^{n} \gamma_{i} x_{i}\right) y_{1}=0$ and, due to $(S), y_{1}=0$. Thus the problem is reduced 
to the case $v \leqq n-1$; and if $\sum \alpha_{i k} x_{i}=0, \sum \alpha_{i k} y_{k}=y_{i}$ for $i, k \geqq 2$, (3) and (4) hold for $i, k=1, \ldots n$ with $\alpha_{1 k}=\alpha_{i 1}=0$.

Proposition 2 implies the following

Corollary. Let $\mathfrak{A}, \mathfrak{A}_{1}, \mathfrak{A}_{2}$ be $C^{*}$-algebras with unit elements, $\mathfrak{A}_{i} \subset \mathfrak{A}$. If $(C)$ and $(S)$ are fulfilled, $\mathfrak{U}_{1} \vee \mathfrak{U}_{2}$ is isomorphic to $\mathfrak{A}_{1} \odot \mathfrak{A}_{2}$.

Here $\mathfrak{U}_{1} \vee \mathfrak{U}_{2}$ denotes the normed involutive subalgebra of $\mathfrak{A}$ generated by $\mathfrak{U}_{1}$ and $\mathfrak{U}_{2} ; \mathfrak{A}_{1} \odot \mathfrak{U}_{2}$ denotes the direct algebraic product of $\mathfrak{A}_{1}$ and $\mathfrak{A}_{2}$, that is, the set of all formal finite sums $\sum x_{i} \otimes y_{i}$ with

$$
\left(\sum_{i} x_{i} \otimes y_{i}\right)\left(\sum_{j} x_{j}^{\prime} \otimes y_{j}^{\prime}\right)=\sum_{i, j} x_{i} x_{j}^{\prime} \otimes y_{i} y_{j}^{\prime} ; \quad\left(\sum x_{i} \otimes y_{i}\right)^{*}=\sum x_{i}^{*} \otimes y_{i}^{*} .
$$

( $\sum x_{i} y_{i}$ and $\sum x_{i} \otimes y_{i}$ are always finite sums).

The isomorphism is given by $\Phi\left(\sum x_{i} y_{i}\right)=\sum x_{i} \otimes y_{i}$.

We have to show that $\mathfrak{A}_{1}$ and $\mathfrak{H}_{2}$ are algebraically independent [6], that is, if $\left\{x_{i}, i=1, \ldots n\right\}$ and $\left\{y_{j}, j=1, \ldots m\right\}$ are sets of linearly independent elements of $\mathfrak{U}_{1}$ and $\mathfrak{U}_{2}$ respectively, then $\left\{x_{i} y_{j}, i=1, \ldots n\right.$, $j=1, \ldots m\}$ is a linearly independent set in $\mathfrak{A}$. Assume the existence of numbers $x_{i j}$ with $\sum_{i, j} x_{i j} x_{i} y_{j}=0$. Then $\sum_{j} x_{j}^{\prime} y_{j}=0$, with $x_{j}^{\prime}=\sum_{i} x_{i j} x_{i}$. Unless all $x_{j}^{\prime}=0$, there are non-trivial $\alpha_{j k}$ such that $\sum_{k} \alpha_{j k} y_{k}=y_{j}$, which contradicts the linear independence of $\left\{y_{j}\right\}$. Hence $x_{j}^{\prime}=\sum x_{i j} x_{i}=0, j=1, \ldots m$, and because of the linear independence of $\left\{x_{i}\right\}$ we get $x_{i j}=0$.

As one can check easily, algebraic independence of $\mathfrak{A}_{1}$ and $\mathfrak{I}_{2}$ implies that $\mathfrak{I}_{1} \vee \mathfrak{U}_{2}$ and $\mathfrak{U}_{1} \odot \mathfrak{U}_{2}$ are isomorphic (cf. [6]).

\section{III.}

The second essential step in proving Theorem 1 is to establish the continuity of the isomorphism $\Phi$ of $\mathfrak{U}_{1} \vee \mathfrak{U}_{2}$ and $\mathfrak{U}_{1} \odot \mathfrak{U}_{2}$.

We shall use the following notations:

$\mathfrak{A}_{12} \equiv \overline{\mathfrak{A}_{1} \vee \mathfrak{A}_{2}}$ denotes the norm-closure of $\mathfrak{A}_{1} \vee \mathfrak{A}_{2}$, that is, the $C^{*}$-subalgebra of $\mathfrak{U}$ generated by $\mathfrak{A}_{1}$ and $\mathfrak{U}_{2}$.

If we define a norm $\beta$ on $\mathfrak{A}_{1} \odot \mathfrak{U}_{2}$, the completion of $\mathfrak{A}_{1} \odot \mathfrak{A}_{2}$ with respect to this norm is denoted by $\mathfrak{A}_{1} \otimes_{\beta} \mathfrak{A}_{2}$.

Definition 1. $\alpha$-norm $[7,8]$ :

$$
\begin{aligned}
& \left\|\sum_{i=1}^{n} x_{i} \otimes y_{i}\right\|_{\alpha} \\
& =\sup \left\{\frac{\varphi_{1} \otimes \varphi_{2}\left[\left(\sum_{i=1}^{m} a_{i} \otimes b_{i}\right)^{*}\left(\sum_{i=1}^{n} x_{i} \otimes y_{i}\right)^{*}\left(\sum_{i=1}^{n} x_{i} \otimes y_{i}\right)\left(\sum_{i=1}^{m} a_{i} \otimes b_{i}\right)\right]}{\varphi_{1} \otimes \varphi_{2}\left[\left(\sum_{i=1}^{m} a_{i} \otimes b_{i}\right)^{*}\left(\sum_{i=1}^{m} a_{i} \otimes b_{i}\right)\right]}\right\},
\end{aligned}
$$


with $x_{i} \in \mathfrak{U}_{1}, y_{i} \in \mathfrak{U}_{2}$; the supremum is taken over all states $\varphi_{1}$ over $\mathfrak{A}_{1}$, all states $\varphi_{2}$ over $\mathfrak{U}_{2}$ and all $a_{i} \in \mathfrak{A}_{1}, b_{i} \in \mathfrak{U}_{2}$. Furthermore,

$$
\varphi_{1} \otimes \varphi_{2}\left[\left(\sum a_{i} \otimes b_{i}\right)\right]=\sum \varphi_{1}\left(a_{i}\right) \varphi_{2}\left(b_{i}\right) .
$$

If $\mathfrak{A}_{1}$ and $\mathfrak{A}_{2}$ are algebras of operators in a Hilbert space $\mathscr{H}, \mathfrak{A}_{1} \odot \mathfrak{A}_{2}$ is an operator algebra in $\mathscr{H} \otimes \mathscr{H}$. In this case, the $\alpha$-norm is identical with the natural norm in $\mathscr{H} \otimes \mathscr{H}$ (theorem of Wulfsohn [9]).

We want to show that $\Phi$ is continuous with respect to the $\alpha$-norm topology in $\mathfrak{U}_{1} \odot \mathfrak{H}_{2}$. We need some definitions and theorems which can be found in mathematical literature, and which are cited below.

Definition 2 [8]. A norm $\beta$ of $\mathfrak{A}_{1} \odot \mathfrak{A}_{2}$ is called compatible (with the algebraic structure of $\mathfrak{A}_{1} \odot \mathfrak{A}_{2}$ ) if the completion of $\mathfrak{A}_{1} \odot \mathfrak{A}_{2}$ with respect to $\beta$ becomes a $C^{*}$-algebra, and if $\|x \otimes y\|_{\beta} \leqq\|x\|\|y\|$.

Definition $3[10]$. A $B^{*}$-norm means any norm $\|\ldots\|_{\beta}$ satisfying $\left\|u^{*} u\right\|_{\beta}=\|u\|_{\beta}^{2}$ for all $u \in \mathfrak{U}_{1} \odot \mathfrak{A}_{2}$.

Proposition (Okayasu) [10]. Every $B^{*}$-norm on $\mathfrak{A}_{1} \odot \mathfrak{H}_{2}$ is compatible.

Theorem (Takesaki and Okayasu) $[8,10]$. Let $\mathfrak{A}_{1}$ and $\mathfrak{A}_{2}$ be $C^{*}$ algebras. Then the set of all $B^{*}$-norms on $\mathfrak{A}_{1} \odot \mathfrak{H}_{2}$ is a complete lattice under the ordering " $\leqq$ " with the least element $\|\ldots\|_{\alpha}$.

Here $\beta_{1} \leqq \beta_{2}$ means $\|u\|_{\beta_{1}} \leqq\|u\|_{\beta_{2}}$ for all $u \in \mathfrak{A}_{1} \odot \mathfrak{A}_{2}$.

We define

and assert

$$
\left\|\sum x_{i} \otimes y_{i}\right\|_{\beta}=\left\|\sum x_{i} y_{i}\right\|
$$

Lemma 3. The norm $\beta$ defined in (5) is a $B^{*}$-norm on $\mathfrak{A}_{1} \odot \mathfrak{A}_{2}$.

Proof. Because of the isomorphism of $\mathfrak{A}_{1} \vee \mathfrak{A}_{2}$ and $\mathfrak{A}_{1} \odot \mathfrak{A}_{2},(5)$ defines a norm on $\mathfrak{A}_{1} \odot \mathfrak{A}_{2} ;$ and

$$
\begin{aligned}
\left\|\left(\sum x_{i} \otimes y_{i}\right)^{*}\left(\sum x_{i} \otimes y_{i}\right)\right\|_{\beta} & =\left\|\sum_{i, j} x_{i}^{*} x_{j} \otimes y_{i}^{*} y_{j}\right\|_{\beta}=\left\|\sum_{i, j} x_{i}^{*} x_{j} y_{i}^{*} y_{j}\right\| \\
& =\left\|\left(\sum x_{i} y_{i}\right)^{*}\left(\sum x_{i} y_{i}\right)\right\|=\left\|\sum x_{i} y_{i}\right\|^{2}=\left\|\sum x_{i} \otimes y_{i}\right\|_{\beta}^{2},
\end{aligned}
$$

since $\mathfrak{A}_{1} \vee \mathfrak{U}_{2}$ is contained in a $C^{*}$-algebra $\mathfrak{U}_{12}$.

Hence $\beta$ is compatible, and, according to the theorem of Takesaki and Okayasu, we have

$$
\left\|\sum x_{i} \otimes y_{i}\right\|_{\alpha} \leqq\left\|\sum x_{i} \otimes y_{i}\right\|_{\beta}=\left\|\sum x_{i} y_{i}\right\| .
$$

The isomorphism $\Phi$ can then be extended to a morphism

$$
\bar{\Phi}: \mathfrak{U}_{12}=\overline{\mathfrak{A}_{1} \vee \mathfrak{A}_{2}} \rightarrow \mathfrak{U}_{1} \otimes_{\alpha} \mathfrak{U}_{2} .
$$

Actually, $\bar{\Phi}$ is a homomorphism because it is surjective: for $\bar{\Phi}\left(\mathfrak{A}_{12}\right)$ is closed ([4], Corollary 1.3.3) and contains $\mathfrak{A}_{1} \odot \mathfrak{A}_{2}$ which is dense in $\mathfrak{A}_{1} \otimes_{\alpha} \mathfrak{I}_{2}$. 
We collect our results formulating

Theorem 2. Let $\mathfrak{A}, \mathfrak{A}_{1}, \mathfrak{A}_{2}$ be $C^{*}$-algebras with unit elements, $\mathfrak{A}_{i} \subset \mathfrak{A}$. Assume

(C) $\mathfrak{U}_{1}$ and $\mathfrak{U}_{2}$ commute elementwise.

(S) If $x$ and $y$ are non-vanishing elements of $\mathfrak{U}_{1}$ and $\mathfrak{U}_{2}$ respectively, then $x y \neq 0$.

Then we have

1) There exists an isomorphism $\Phi: \mathfrak{U}_{1} \vee \mathfrak{U}_{2} \rightarrow \mathfrak{U}_{1} \odot \mathfrak{U}_{2}$.

2) $\Phi$ is continuous with respect to the $\alpha$-norm on $\mathfrak{H}_{1} \odot \mathfrak{A}_{2}$ and can therefore be extended to a homomorphism $\bar{\Phi}: \mathfrak{A}_{12} \rightarrow \mathfrak{U}_{1} \otimes_{\alpha} \mathfrak{A}_{2}$.

3) Let $\mathfrak{M}$ be any abelian $C^{*}$-subalgebra of $\mathfrak{A}_{1}$. The restriction of $\bar{\Phi}$ to $\overline{\mathfrak{M} \vee \mathfrak{U}_{2}}$ is an isomorphism, $\bar{\Phi}\left(\overline{\mathfrak{M} \vee \mathfrak{U}_{2}}\right)=\mathfrak{M} \otimes_{\alpha} \mathfrak{H}_{2}$.

Parts 1) and 2) are proven. The third part follows from another theorem of Takesaki:

Theorem (Takesaki) [8]. Let $\mathfrak{A}_{1}$ be an abelian $C^{*}$-algebra. Then, for any $C^{*}$-algebra $\mathfrak{A}_{2}$, the $\alpha$-norm is the only compatible norm on $\mathfrak{A}_{1} \odot \mathfrak{A}_{2}$. Therefore, since we know that the norm $\beta$ defined in (5) is compatible, we have for $x_{i} \in \mathfrak{M}$

$$
\left\|\sum x_{i} \otimes y_{i}\right\|_{\alpha}=\left\|\sum x_{i} \otimes y_{i}\right\|_{\beta}=\left\|\sum x_{i} y_{i}\right\| ;
$$

and this implies that the restriction of $\bar{\Phi}$ to $\overline{\mathfrak{M} \vee \mathfrak{A}_{2}}$ is an isomorphism of $\overline{\mathfrak{M} \vee \mathfrak{U}_{2}}$ and $\mathfrak{M} \otimes_{\alpha} \mathfrak{U}_{2}$.

This completes the proof of Theorem 2.

IV.

Finally, we shall prove Theorem 1 and Proposition 1. As already mentioned, Schlieder [2] showed that $(\mathrm{S})$ is a necessary condition. (The proof given in [2] is not a quite general one, for one needs the existence of sufficiently many hermitian elements $x \in \mathfrak{A}_{1}$ and $y \in \mathfrak{A}_{2}$ with $x^{2}=x$, $y^{2}=y$; its generalization is given in the appendix.)

Now let us assume that $(S)$ is satisfied; so we can use theorem 2. Let $\tilde{\varphi}$ be any continuous linear functional over $\mathfrak{A}_{1} \otimes_{\alpha} \mathfrak{H}_{2}$. Then we define a linear functional $\varphi$ over $\mathfrak{A}_{12}$ by

$$
\varphi(u)=\tilde{\varphi}(\bar{\Phi}(u)), \quad u \in \mathfrak{A}_{12} ; \quad \text { in short }: \varphi=\tilde{\varphi} \circ \bar{\Phi} .
$$

$\bar{\Phi}$ is continuous; therefore, $\varphi$ is continuous. Clearly, if $\tilde{\varphi}$ is positive, so is $\varphi$, since $u \geqq 0, u \in \mathfrak{A}_{12}$, implies $\bar{\Phi}(u) \geqq 0$. Put $\tilde{\varphi}=\varphi_{1} \otimes \varphi_{2}, \varphi_{1}$ and $\varphi_{2}$ arbitrary states over $\mathfrak{A}_{1}$ and $\mathfrak{I}_{2}$ respectively, then

$$
\varphi=\varphi_{1} \otimes \varphi_{2} \circ \bar{\Phi}
$$


is the functional over $\mathfrak{U}_{12}$ required by statistical independence:

$$
\begin{aligned}
& x \in \mathfrak{A}_{1}: \varphi(x)=\tilde{\varphi}(\bar{\Phi}(x))=\tilde{\varphi}(x \otimes \mathbf{1})=\varphi_{1}(x) ; \\
& y \in \mathfrak{A}_{2}: \varphi(y)=\tilde{\varphi}(\bar{\Phi}(y))=\tilde{\varphi}(\mathbf{1} \otimes y)=\varphi_{2}(y) .
\end{aligned}
$$

It remains to be checked whether $\varphi_{1} \otimes \varphi_{2}$ is continuous and positive if $\varphi_{1}$ and $\varphi_{2}$ are continuous and positive. The continuity is a direct consequence of the Definition 1 of the $\alpha$-norm; the positivity follows from an easily provable lemma:

Lemma 4 [6]. If $\varphi_{1}$ and $\varphi_{2}$ are positive functionals over $\mathfrak{A}_{1}$ and $\mathfrak{A}_{2}$ respectively, then $\varphi_{1} \otimes \varphi_{2}$ is a positive functional over $\mathfrak{A}_{1} \odot \mathfrak{U}_{2}$.

Because of the continuity, $\varphi_{1} \otimes \varphi_{2}$ is also positive over $\mathfrak{A}_{1} \otimes_{\alpha} \mathfrak{H}_{2}$. This proves the statistical independence of $\mathfrak{A}_{1}$ and $\mathfrak{H}_{2}$, since the state $\varphi$ over $\mathfrak{U}_{12}$ defined in (8) can be extended to a state over $\mathfrak{\mathfrak { U }}$. We note that

$$
\varphi(x y)=\varphi_{1}(x) \varphi_{2}(y)=\varphi(x) \varphi(y), \quad x \in \mathfrak{A}_{1}, \quad y \in \mathfrak{A}_{2} .
$$

Proof of Proposition 1. Let $\mathscr{E}(\mathfrak{2})$ denote the set of states over $\mathfrak{A}$ and $\mathscr{P}(\mathfrak{A})$ the subset of pure states. If $\varphi_{1}$ and $\varphi_{2}$ are pure states, they define irreducible representations $\pi_{\varphi_{1}}$ and $\pi_{\varphi_{2}}$ of $\mathfrak{A}_{1}$ and $\mathfrak{H}_{2}$ respectively. The representation $\pi$ of $\mathfrak{I}_{1} \otimes_{\alpha} \mathfrak{A}_{2}$, defined by $\varphi_{1} \otimes \varphi_{2}$ is isomorphic to $\pi_{\varphi_{1}}\left(\mathfrak{U}_{1}\right) \otimes \pi_{\varphi_{2}}\left(\mathfrak{A}_{2}\right)$, therefore, $\pi$ is irreducible and $\varphi_{1} \otimes \varphi_{2} \in \mathscr{P}\left(\mathfrak{U}_{1} \otimes_{\alpha} \mathfrak{H}_{2}\right)$.

According to Theorem 2, $\mathfrak{A}_{12} / \operatorname{Ker} \bar{\Phi}$ and $\mathfrak{I}_{1} \otimes_{\alpha} \mathfrak{H}_{2}$ are isomorphic; so $\tilde{\varphi} \rightarrow \tilde{\varphi} \circ \bar{\Phi}$ defines an isomorphism $\Phi^{\prime}$ of $\mathscr{E}\left(\mathfrak{A}_{1} \otimes_{\alpha} \mathfrak{A}_{2}\right)$ and $\mathscr{E}\left(\mathfrak{U}_{12} / \operatorname{Ker} \bar{\Phi}\right)$, which transforms pure states into pure states. Therefore, $\varphi=\varphi_{1} \otimes \varphi_{2} \circ \bar{\Phi}$ is an element of $\mathscr{P}\left(\mathfrak{A}_{12} / \operatorname{Ker} \bar{\Phi}\right)$. (Here we identify $\mathscr{E}\left(\mathfrak{A}_{12} / \operatorname{Ker} \bar{\Phi}\right)$ with the set $\mathscr{E}_{0}=\left\{\chi \mid \chi \in \mathscr{E}\left(\mathfrak{U}_{12}\right), \chi(\operatorname{Ker} \bar{\Phi})=0\right\}$.) Now consider $\varphi$ as a state over $\mathfrak{H}_{12}$ and suppose that $\varphi$ majorizes a state $\varphi^{\prime} \in \mathscr{P}\left(\mathfrak{H}_{12}\right)$. Since $\varphi(x)=0$ for all $x \in \operatorname{Ker} \bar{\Phi}$, the same holds for $\varphi^{\prime}$, which implies $\varphi^{\prime} \in \mathscr{P}\left(\mathfrak{A}_{12} / \operatorname{Ker} \bar{\Phi}\right)$. But this is a contradiction unless $\varphi^{\prime}=\varphi$; and therefore, $\varphi \in \mathscr{P}\left(\mathfrak{A}_{12}\right)$. Any pure state over $\mathfrak{U}_{12}$ can be extended to a pure state over $\mathfrak{A}$; which completes the proof.

Acknowledgement. This work was suggested by Prof. Borchers whom I wish to express my thanks for continuing encouragement and many helpful discussions.

\section{Appendix}

Let $\mathfrak{U}_{1}, \mathfrak{H}_{2}$ be commuting $C^{*}$-algebras with unit elements, $\mathfrak{U}_{i} \subset \mathfrak{U}$, and let $\mathfrak{A}_{1}$ and $\mathfrak{U}_{2}$ be statistically independent. We want to show that $x y \neq 0$ whenever $x \in \mathfrak{A}_{1}, y \in \mathfrak{H}_{2}, x$ and $y \neq 0$.

Assume that we can find non-vanishing elements $x^{\prime} \in \mathfrak{U}_{1}$ and $y^{\prime} \in \mathfrak{U}_{2}$ with $x^{\prime} y^{\prime}=0$. Then of course $x^{\prime *} x^{\prime} y^{*} y^{\prime}=0$. Let $\alpha \in \operatorname{Sp}\left(x^{\prime *} x^{\prime}\right), \alpha \neq 0$ (Sp $u$ denotes the spectrum of $u$ in $\mathfrak{A}_{1}$ ). Then for $x=\alpha^{-1} x^{*} x \in \mathfrak{A}_{1}$, 
$y=y^{*} y^{\prime} \in \mathfrak{A}_{2}$, we have

$$
\begin{gathered}
x y=0, \quad x \neq 0, \quad y \neq 0, \\
x^{*}=x, \quad 1 \in \operatorname{Sp} x,
\end{gathered}
$$

and therefore,

$$
z \equiv(1-x)^{2} \geqq 0, \quad 0 \in \mathrm{Sp} z, \quad \alpha \in \mathrm{Sp}(z+\alpha) .
$$

Consider the selfadjoint vector space $\mathscr{D}$ spanned by $\{1, z\}$ and define $\varphi_{1}(\mathbf{1})=1, \varphi_{1}(z)=0 . \varphi_{1}$ is a positive functional on $\mathscr{D}$ because, according to (iii), $\gamma_{1} \cdot \mathbf{1}+\gamma_{2} z \geqq 0$ implies $\gamma_{1} / \gamma_{2} \geqq 0$ if $\gamma_{2} \neq 0$, hence, $\varphi_{1}\left(\gamma_{1} \mathbf{1}+\gamma_{2} z\right)$ $=\gamma_{1} \geqq 0$. As is well known (cf. [4], Lemma 2.10.1), $\varphi_{1}$ can be extended to a state over $\mathfrak{U}_{1}$, and we have

$$
\varphi_{1}\left((1-x)^{2}\right)=0
$$

and because of $\left|\varphi_{1}(u)\right|^{2} \leqq\left\|\varphi_{1}\right\| \varphi_{1}\left(u^{*} u\right)$ :

$$
\varphi_{1}(1-x)=0 .
$$

It is clear that we can find a state $\varphi_{2}$ over $\mathfrak{A}_{2}$ with $\varphi_{2}(y) \neq 0$.

Since $\mathfrak{A}_{1}$ and $\mathfrak{U}_{2}$ are statistically independent, there exists a common extension $\varphi$ of $\varphi_{1}$ and $\varphi_{2}$. The Schwartz inequality implies

$$
|\varphi((1-x)(1+y))|^{2} \leqq \varphi\left((1-x)^{2}\right) \varphi\left((1+y)^{2}\right)=\varphi_{1}\left((1-x)^{2}\right) \varphi_{2}\left((1+y)^{2}\right) .
$$

Hence, according to (iv), $\varphi((1-x)(1+y))=0$. However,

$$
\varphi((1-x)(1+y))=\varphi(1-x+y)=\varphi_{1}(1-x)+\varphi_{2}(y)=\varphi_{2}(y) \neq 0
$$

according to (i) and (v), which is a contradiction.

\section{References}

1. Haag, R., Kastler, D.: J. Math. Phys. 5, 848 (1964).

2. Schlieder, S.: Commun. Math. Phys. 13, 216 (1969).

3. Rickart, Charles E.: General theory of Banach algebras. Princeton: Van Nostrand 1960.

4. Dixmier, Jaques: Les $C^{*}$-algèbres et leurs représentations. Paris:Gauthier-Villars 1964.

5. The original version of the proof of lemma 1 needed the assumption that there exists a representation $\pi$ of $\mathfrak{U}_{12}$ with $\pi\left(\mathfrak{U}_{1}\right)^{\prime \prime}$ and $\pi\left(\mathfrak{U}_{2}\right)^{\prime \prime}$ fulfilling the proposition of Schlieder. The idea of the proof given in this paper is due to Borchers.

6. Turumaru, T.: Tôhoku Math. J. 8, 281 (1956).

7. - Tôhoku Math. J. 4, 242 (1952).

8. Takesaki, M.: Tôhoku Math. J. 16, 111 (1964).

9. Wulfsohn, A.: Bull. Sci. Math. 87, 13 (1963).

10. Okayasu, T.: Tôhoku Math. J. 18, 325 (1966).

H. Roos

Institut $\mathrm{f}$. theoret. Physik der Universität 3400 Göttingen,

Bunsenstraße 9 